Short Report

\title{
Field evaluation of a commercially available COVID-19 rapid antigen test kit
}

\author{
VR Francis, MARV Muthugala \\ Sri Lankan Journal of Infectious Diseases 2021 Vol.11(1):13-17 \\ DOI: http://dx.doi.org/10.4038/sljid.v11i1.8356
}

\begin{abstract}
Filtering out of infectious individuals to interrupt the chain of transmission is the major strategy for control of COVID-19 pandemic. Rapid antigen testing is a useful tool to achieve this purpose. We evaluated a commercial rapid antigen test kit (STANDARD Q COVID-19 Ag test, SD Biosensor, Republic of Korea) which was supplied by the Ministry of health, Sri Lanka for COVID -19 testing, at field level in the Eastern Province of Sri Lanka.
\end{abstract}

We tested 40 known COVID-19 positive asymptomatic persons and 143 asymptomatic vendors working in a congested public market, regarded as a high-risk location using STANDARD Q COVID-19 Ag test and the gold standard real-time RT-PCR on simultaneous samples according to the manufacturers' instructions.

The overall sensitivity of the STANDARD Q COVID-19 Ag kit was 58.5\% (95\% CI: 44,72 ) which increased to $79.4 \%$ in people with high viral load $(\mathrm{Ct}<25)$. Specificity of this kit. in our study was $100 \%$.

We recommend rapid antigen test as a primary screening test for community surveillance as it is a cost-effective measure of pandemic control.

Keywords: Rapid antigen test, COVID -19, RT PCR, COVID -19 community surveillance, field evaluation.

\section{Introduction}

Rapid identification and isolation of infectious individuals are a key preventive measure during the COVID-19 pandemic. Real-time reverse transcription polymerase chain reaction (rRT-PCR)

\footnotetext{
${ }^{1}$ Faculty of Health-Care Sciences, Eastern University, Sri Lanka

${ }^{2}$ National Hospital, Kandy, Sri Lanka

Address for correspondence: Dr.V.R.Francis Faculty of Health-Care Sciences, Eastern University, Sri Lanka.

Telephone no: +94773642462 E-mail: vaithehir@esn.ac.lk

\section{https://orcid.org/0000-0003-1854-0796}

Received 8 March 2021 and revised version accepted 29 March 2021

(c) (i) This an open-access article distributed under the terms of the Creative Commons Attribution License, which permits unrestricted use, distribution, and reproduction in any medium, provided the original author and source are credited.
} 
assays were used by laboratories around the world to detect SARS-Cov-2 virus which is the causative agent of COVID-19. However, the cost and availability of rRT-PCR is challenging in many countries. The development of a rapid, cheap and reliable point of care test is therefore considered a major milestone in reducing the impact of the pandemic. ${ }^{1}$

Evaluation of a test kit at local settings is important prior to the wide spread use of a test kit. Therefore, we aimed to evaluate a commercially available rapid antigen test kit, STANDARD Q COVID-19 Ag Test (SD Biosensor, Republic of Korea) which was issued by the medical supplies division of the Ministry of Health since mid-November 2020 to the major hospitals in Sri Lanka and to the regional epidemiologists.

STANDARD Q COVID-19 Ag Test is a rapid chromatographic immunoassay for the qualitative detection of specific antigens of SARS-CoV-2 present in the human nasopharynx. ${ }^{2}$

\section{Objectives:}

To evaluate the performance of the rapid antigen test kit against the $\mathrm{Ct}$ values of real-time RTPCR testing, using simultaneously taken samples.

\section{Methods}

We tested 40 newly diagnosed asymptomatic SARS-CoV-2 PCR positive individuals admitted to the Kathankudy and Karadiyanaru COVID intermediate care centers during November 2020, using the Standard Q COVID-19 Ag test kit (SD Biosensor, Republic of Korea). We also performed field testing of this rapid antigen test kit on 143 asymptomatic vendors at Akkaraipatthu market area on the $26^{\text {th }}$ of November 2020. Akkaraipatthu market is a busy and congested local market. We performed the rapid antigen test on nasopharyngeal swabs according to the manufacturer's instructions. Simultaneously we collected mid-nasal and throat swabs in $3 \mathrm{ml}$ of viral transport medium for real-time RT-PCR assay. The real-time RT-PCR assay was performed on the same day at the COVID-19 diagnostic laboratory of the Teaching Hospital, Batticaloa. Extraction of viral RNA was performed using the SpinStar ${ }^{\mathrm{TM}}$ Viral Nucleic acid extractor kit 1.0 (ADT Biotech, Malaysia) and real-time RT-PCR assay was performed using the RealStar ${ }^{\circledR}$ SARS-CoV-2 RT-PCR Kit 1.0 (Altona diagnostics, Germany) according to the manufacturer's instructions. All the reagents and consumables were provided by the Medical supplies division of the Ministry of Health.

Collection of samples and antigen testing in the field were performed by trained public health officers and Medical laboratory technologists, respectively. Samples in lysis buffer were added to the antigen strips as five strips at a time. The added time and reading time were recorded for each batch (5 strips per batch) of strips. The strips were read according to the manufacturer's instructions. 


\section{Results}

Fifty three of 183 samples were positive on RT PCR testing and 127 samples negative (Table 1). Three samples gave inconclusive results on RT PCR as only one gene (either E gene or S gene) was detected All 183 samples showed valid antigen test results. Thirty one of 183 samples had a positive antigen test and the remaining were antigen negative. We noticed that the "Test" line appeared first while the positive samples moved along the strips towards the "Control" line. On all positive strips, when the "Control line" was visible the "Test" line was clearly visible too.

Table 1: Results of Covid-19 Ag test and RT PCR test

\begin{tabular}{llcccc}
\hline & & \multicolumn{3}{c}{ PCR } & \\
\cline { 3 - 5 } & & Positive & Negative & Inconclusive & Total \\
\hline \multirow{2}{*}{ Ag } & Positive & $\mathbf{3 1}$ & $\mathbf{0}$ & 0 & 31 \\
& Negative & 22 & 127 & 3 & 152 \\
\multicolumn{2}{l}{ Tnconclusive } & 0 & 0 & 0 & 0 \\
\multicolumn{2}{l}{ Total } & $\mathbf{5 3}$ & $\mathbf{1 2 7}$ & 3 & $\mathbf{1 8 3}$ \\
\hline
\end{tabular}

32, $8(38 \%)$ gave a positive rapid antigen test (Table 2).
Twenty-nine positive samples had real-time RTPCR Ct value less than 25 . Twenty three of 29 samples (79\%) which had a $\mathrm{Ct}$ value of $<25$ were rapid antigen positive. Of 21 samples which showed $\mathrm{Ct}$ values between 25 to

Table 2: Rapid antigen test positivity and real-time RT-PCR Ct value.

\begin{tabular}{lccc}
\hline $\mathbf{C t}$ & $\mathbf{2 5}$ & $\mathbf{2 5}-\mathbf{3 2}$ & $>\mathbf{3 2}$ \\
\hline Ag Positive & $\mathbf{2 3}(\mathbf{7 9 \%})$ & $8(38 \%)$ & 0 \\
Ag Negative & 6 & 13 & 6 \\
Total & $\mathbf{2 9}$ & 21 & 6 \\
\hline
\end{tabular}

Not a single sample negative on rRT-PCR was positive with the antigen test.

\section{Discussion}

STANDARD Q COVID-19 Ag test (SD Biosensor, Republic of Korea) is a user-friendly test method with an all-inclusive test kit; therefore, it is an easy to perform test at field level.

The major concern here is that this rapid antigen test kit failed to detect 20.6\% (6 out of 29) and $61 \%$ (13 out of 21) of samples with real-time RT-PCR Ct values $<25$ and between 25 and 32 respectively. However, if the test is used repeatedly on populations where risk of transmission is high (e.g. Market workers), the number of missing cases can be minimized.

The overall sensitivity of STANDARD Q COVID-19 Ag test in our study is $58.5 \%$ (95\% CI: 44, 72) against the real-time RT PCR. The sensitivity increases to 79.4\% (95\% CI: 60, 92) when samples are with real-time RT-PCR Ct $<25$. It indicates that the STANDARD Q COVID-19 Ag test performs better when the viral load is high. A similar finding was observed in a meta-analysis 
of rapid antigen tests done by European Centre for Disease Control and Prevention. ${ }^{3}$ Interpretation of sensitivity of a point of care testing should be done considering the purpose of testing. Point-of care testing in COVID-19 is aimed to break the chain of transmission by detecting and isolating highly infectious individuals. ${ }^{4}$ The turnaround time of a test is a key factor to achieve successful containment. Repeated testing on population with increased risk of transmission could be an effective strategy to prevent institutional outbreaks noted in the $2^{\text {nd }}$ wave of COVID-19 in Sri Lanka. Tests with longer turnaround time with higher cost like real-time RT-PCR will not be useful for repeated testing. ${ }^{4}$

There are several issues in considering the real-time RT-PCR as the gold standard for measuring the sensitivity of rapid antigen tests. One important area of concern is that individuals can show positive real-time RT-PCR results for several weeks without evidence of active viral replication. ${ }^{4}$

Specificity of STANDARD Q COVID-19 Ag test in our study is $100 \%$ as there are no false positive results. The specificity of rapid antigen test varies from $80 \%$ to $100 \%$ in other studies. ${ }^{5,6,7}$ An Australian study conducted at a low prevalence setting has also revealed high specificity of antigen-based SARS-Cov-2 testing. ${ }^{8}$ However, the study recommends confirming all positive SARS-Cov-2 antigen-based tests in a low prevalence setting with real-time RTPCR. ${ }^{8}$ The prevalence of COVID-19 in our study population has been calculated as $29 \%$ according to the real-time RT-PCR results.

There were no invalid results using the STANDARD Q COVID-19 Ag test in our study and therefore the kit's failure rate is $0 \%$. The positive and negative predictive values were $100 \%$ (95\% CI: $0.97,1.00)$ and $85 \%(95 \%$ CI: $0.79,0.91)$ respectively in our study.

\section{Conclusion}

STANDARD Q COVID-19 Ag test shows satisfactory performance with $100 \%$ specificity and $79 \%$ sensitivity in asymptomatic people with high viral load in our study. As it is a point-of-care test, it has several advantages over the real-time RT-PCR assays in terms of earlier results, ease of use and in usability in field testing. Therefore, it can be used effectively to filter out the infectious individuals in high-risk communities to interrupt the chain of transmission.

Conflict of interest: The authors declare no conflict of interests.

Ethical clearance: STANDARD Q COVID-19 Ag test was introduced as part of the COVID - 19 testing process by the Ministry of Health, Sri Lanka and we did the testing as a part of community screening in the Eastern province.

\section{Acknowledgement:}

We wish to extend our gratitude to the Medical laboratory technologists attached to the Teaching Hospital Batticaloa, Mr.G.Arunprasad, Mrs.Dinusha Priyanganie and Mrs.Jeewani Mel who voluntarily participated at the field testing at Akkaraipatthu. We would also like to express our special thanks to Mr.S.Ravishankar, chief Medical laboratory technologist of Covid PCR 
laboratory of Teaching Hospital Batticaloa for arranging all the necessary logistics for field testing as well as for the PCR testing.

We wish to express our sincere thanks to all staff of BH Kathankudy, DH Karadianaru, Regional Epidemiologist, Kalmunai, RDHS Kalumnai and their team for their kind support and all the patients who provided the samples for the evaluation.

We sincerely acknowledge Mr. S. Santharooban, Senior Assistant Librarian of Faculty of HealthCare Sciences, Eastern University, Sri Lanka, for his assistance in statistical analysis of our data.

\section{Contribution of authors}

VR formulated the study and provided direct supervision of field and laboratory testing. MARV supported formulation of the study design and provided expert support for RT PCR testing and interpretation.

\section{References}

1. World Health Organization. (2020). SARS-CoV-2 antigen-detecting rapid diagnostic tests: an implementation guide. World Health Organization. Available at: https://apps.who.int/iris/handle/10665/337948. License: CC BY-NC-SA 3.0 IGO

2. STANDARD ${ }^{\mathrm{TM}} \mathrm{Q}$ COVID - 19 Ag Test (Package insert). Republic of Korea: SD Biosensor Inc.: 2020.

3. European Centre for Disease Prevention and Control. Options for the use of rapid antigen tests for COVID-19 in the EU/EEA and the UK. 19 November 2020. ECDC: Stockholm; 2020.

Available at: https://www.ecdc.europa.eu/en/publications-data/options-use-rapid-antigen-testscovid-19-eueea-and-uk

4. Mina, M.J, Parker, R. Larremore, D.B, Rethinking Covid-19 test sensitivity - a strategy for containment. $N$ Engl J Med. 2020; 383:e120. https://doi.org/10.1056/NEJMp2025631

5. Nalumansi, T. Lutalo, J. Kayiwa et al. Field evaluation of the performance of a SARS-CoV-2 antigen rapid diagnostic test in Uganda using nasopharyngeal samples. Int J of Infectious Diseases 2021; 104:282-286. doi: https://doi.org/10.1016/j.ijid.2020.10.0731

6. Albert, Eliseo et al. Field evaluation of a rapid antigen test (Panbio ${ }^{\mathrm{TM}}$ COVID-19 Ag Rapid Test Device) for COVID-19 diagnosis in primary healthcare centres. Clinical microbiology and infection: the official publication of the European Society of Clinical Microbiology and Infectious Diseases 2021; 27(3):472.e7-472.e10. doi:10.1016/j.cmi.2020.11.004

7. Preliminary report from the Joint PHE Porton Down \& University of Oxford SARS-CoV-2 test development and validation cell: rapid evaluation of lateral flow viral antigen detection devices (LFDs) for mass community testing. $8^{\text {th }}$ Nov 2020.

Available at:

https://www.ox.ac.uk/sites/files/oxford/media_wysiwyg/UK\%20evaluation_PHE\%20Porton\%20D own\%20\%20University\%20of\%200xford_final.pdf.

8. S. Muhi, N. Tayler, T. Hoang et al The Lancet Regional Health - Western Pacific 2021; 9: 10011

https://doi.org/10.1016/j.lanwpc.2021.100115. 